\title{
Mündlichkeit und Schriftlichkeit im schulischen Deutschunterricht in England
}

\section{Document Version}

Accepted author manuscript

Link to publication record in Manchester Research Explorer

\section{Citation for published version (APA):}

Durrell, M. (2017). Mündlichkeit und Schriftlichkeit im schulischen Deutschunterricht in England. Deutsch als Fremdsprache, 54(3), 131-141. https://www.dafdigital.de/ce/muendlichkeit-und-schriftlichkeit-im-schulischendeutschunterricht-in-england/detail.html

\section{Published in:}

Deutsch als Fremdsprache

\section{Citing this paper}

Please note that where the full-text provided on Manchester Research Explorer is the Author Accepted Manuscript or Proof version this may differ from the final Published version. If citing, it is advised that you check and use the publisher's definitive version.

\section{General rights}

Copyright and moral rights for the publications made accessible in the Research Explorer are retained by the authors and/or other copyright owners and it is a condition of accessing publications that users recognise and abide by the legal requirements associated with these rights.

\section{Takedown policy}

If you believe that this document breaches copyright please refer to the University of Manchester's Takedown Procedures [http://man.ac.uk/04Y6Bo] or contact uml.scholarlycommunications@manchester.ac.uk providing relevant details, so we can investigate your claim.

\section{OPEN ACCESS}


Martin Durrell

Mündlichkeit und Schriftlichkeit im schulischen

Deutschunterricht in England ${ }^{1}$

In diesem Beitrag wird auf der Basis von Befragungen mit Studierenden und Lehrenden an englischen Schulen und an der Universität Manchester über das Verhältnis von Mündlichkeit und Schriftlichkeit beim Deutschunterricht an englischen Schulen berichtet. Die Struktur der vom Kultusministerium begutachteten Lehrpläne sowie der Anlass zu den Reformen, die gegenwärtig im Gang sind, werden dargestellt. In der Oberstufe (für das A-level Examen zur Hochschulreife) wird Deutsch im Rahmen von festgelegten Themen zu Gesellschaft, Geschichte, Leben und Kultur des deutschsprachigen Raumes unter Verwendung von authentischem Material unterrichtet. Dabei wird aber der progressive Erwerb von Grammatik und Wortschatz nicht systematisch beachtet, so dass die erworbenen Kenntnisse eher fragmentarisch und vorwiegend auf die Ansprüche der Prüfungen gerichtet sind. Vor allem merkt man einen ausschließlichen Fokus auf die konzeptionelle Schriftlichkeit, auch beim Unterricht im Hörverständnis und in der Sprechfertigkeit, so dass es den Absolventen des Lehrplans an echter Kompetenz (sowie auch an Selbstvertrauen) im Umgang mit der spontanen informellen Mündlichkeit von Muttersprachlern fehlt.

The present paper deals with the relationship between spoken and written language in teaching German in English schools, on the basis of enquiries and interviews with teachers and pupils at a number of schools and colleges, and at the University of Manchester. We explain the structure of the curriculum, as determined ultimately by the Ministry for Education and its departments, and consider the motivations 
for ongoing reforms of the school syllabus. In the last two years of secondary school German is taught for the A-level examinations on the basis of prescribed topics about the life, culture, society and history of the German-speaking countries on the basis of authentic material. However, no systematic attention is paid to the acquisition of elements of grammar or vocabulary, so that knowledge of these can be relatively limited to what is necessary to succeed in the examination. Above all, there is an exclusive concentration on the written language, with the acquisition of competence in listening and speaking also based on texts in formal registers, so that students completing the A-level programmes lack the competence (or even confidence) to cope with the register of informal colloquial speech as used spontaneously by native speakers in everyday communication.

\section{Zur Untersuchungsgrundlage}

Für den vorliegendenBeitrag zum Themenschwerpunkt „Mündlichkeit im DaF-Unterricht“" soll hier im Anschluss an frühere Arbeiten zu diesem Thema (zuletzt Durrell 2012) untersucht werden, inwieweit Registerunterschiede im schulischen DaF-Unterricht in England beachtet werden insbesondere die Unterschiede zwischen den präskriptiven, als „korrekt“ geltenden Normen der kodifizierten Standardsprache, die für den schriftlichen Gebrauch verbindlich sind, und den oft davon abweichenden umgangssprachlichen Gebrauchsnormen, die für die informelle mündliche Kommunikation charakteristisch sind. Trotz der „kommunikativen Wende“ in der Praxis des Fremdsprachenunterrichts (vgl. z.B. Ellis / Shitani 2014: 4349 und Rösler 2016: 138) und der Abkehr von der herkömmlichen Grammatik-Übersetzungsmethode in den letzten Jahrzehnten berichten Studienanfänger der 
Germanistik an englischen Hochschulen seit mehreren Jahren, dass sie sich im Umgang mit dem alltäglich gesprochenen Deutsch häufig unsicher fühlen, obwohl ihre Kompetenzen den Ansprüchen der englischen Prüfungsbehörden für die Zulassung zum Universitätsstudium im Fach Deutsch durchaus genügen.

Um diesem scheinbaren Gegensatz nachzugehen, wurden im Herbst 2016 Befragungen unternommen bei Studienanfängern der Germanistik an der Universität Manchester sowie bei Lektorinnen und Kolleginnen des Germanistischen Instituts, auch wurden Beobachtungen unternommen bei sprachpraktischen Übungen. Des Weiteren wurden längere Interviews mit zehn Deutschlehrerinnen an Schulen in Nordwestengland durchgeführt, die ihre Schüler auf die offiziellen Abschlussprüfungen vorbereiten, sowie auch fünf Sekundarschulen bzw. Oberstufenkollegs besucht. Auch dort wurde Lehrveranstaltungen beigewohnt. ${ }^{2}$ Darüber hinaus wurden mit drei KollegInnen Gespräche geführt, die an der Neugestaltung der englischen Reifeprüfungen in modernen Fremdsprachen teilnahmen, die im Abschnitt 7 unten ausführlicher besprochen werden ${ }^{3}$. In den Interviews mit den Lernenden wurde auf Grund einer kurzen Frageliste festgestellt, inwieweit sie mit charakteristischen Erscheinungen der Mündlichkeit vertraut waren, z.B. reduzierte Formen der Artikel und Pronomina, Apokope in der Verbmorphologie, Ausklammerung, sowie typische umgangssprachliche Lexis, und entsprechend wurde in den beobachteten Unterrichtsstunden darauf geachtet, ob Lehrende und Lernende solche Formen verwendeten oder eher diejenigen, die für die konzeptuelle Schriftlichkeit typisch sind und im Großen und Ganzen den präskriptiven hochsprachlichen Normen entsprechen. Die Lehrerinnen wurden dann gefragt, ob sie die Lernenden systematisch (oder überhaupt) auf Registerunterschiede aufmerksam 
machten (z.B. auf den Unterschied zwischen dem (betonten) Zahlwort ein und dem (unbetonten) unbestimmten Artikel 'n) bzw. ob sie den Lernenden vom Gebrauch von Formen abrieten, die von der kodifizierten hochsprachlichen Norm abwichen, weil solche in der mündlichen Prüfung negativ bewertet werden könnten.

Die folgenden Ausführungen fußen auf den Ergebnissen dieser Befragungen, wobei betont werden muss, dass diese nur als ein erster, relativ beschränkter Einblick in einen sehr breiten Problemkomplex gelten dürfen und kaum den Anspruch einer systematischen Repräsentativität stellen können. Es kann jedoch festgehalten werden, dass die Ansichten aller Beteiligten und die Ergebnisse der Befragungen bemerkenswert einheitlich waren, was vielleicht auf die im Folgenden explizierte Struktur der nationalen Prüfungen zurückzuführen ist. Nur in einigen wenigen später zu erörternden Fällen traten signifikante Unterschiede bei den Antworten auf. Auch stimmen diese Befunde mit den eigenen Beobachtungen von Studienanfängern im Fach Germanistik in den letzten zwanzig Jahren weitgehend überein.

\section{Das englische Schulwesen und der}

\section{Fremdsprachenunterricht}

Zum Verständnis des Problems ist eine kurze

Beschreibung der Stellung von Fremdsprachen im englischen Schulwesen nötig. Diese ist nach weitreichenden Reformen in den letzten Jahren recht kompliziert, und hier sollen nur ein paar relevante Details skizziert werden.

Nach dem nationalen Lehrplan für englische Schulen (vgl. DfE 2013) ist der Unterricht in einer Fremdsprache in den Klassen 3 bis 5 (sog. Key Stage 2) und den Klassen 6 bis 9 (sog. Key Stage 3) obligatorisch. In den Klassen 10 und 11 (Key Stage 4) ist der Fremdsprachenunterricht ein Wahlfach, bei dem die Schüler auf eine nationale Prüfung zur mittleren 
Reife hin, das General Certificate of Secondary Education $(G C S E)^{4}$ arbeiten. Dafür gibt es einen vom

Kultusministerium in groben Zügen festgelegten Lehrplan, und die Leistungen der Schüler werden durch schriftliche Klausuren (sowie für Fremdsprachen auch eine mündliche Prüfung) bewertet. Detaillierte Lehrpläne werden von unabhängigen Prüfungsbehörden nach den vom Ministerium vergebenen Richtlinien herausgearbeitet, und diese sind auch für die Zusammensetzung und die Korrektur der Prüfungen zuständig. In England bestehen drei solche Prüfungsbehörden ${ }^{5}$, und jede Schulleitung darf für jedes Fach frei zwischen den angebotenen Lehrplänen wählen.

In den letzten Schuljahren, d.h. in den 12. und 13. Klassen, bereiten sich die Schüler auf eine Abschlussprüfung, das General Certificate of Education (GCE) Advanced Level (kurz A-level genannt) vor, die über die Zulassung zum Universitätsstudium bestimmt. Die Lehrgänge für A-levels werden von den anerkannten Prüfungsbehörden nach vom Bildungsministerium festgelegten groben Richtlinien herausgearbeitet. Bis vor kurzem bestanden diese Lehrgänge aus zwei Teilen, dem Advanced Subsidiary level bzw. ASlevel, das am Ende des 12. Schuljahrs abgelegt wurde, und dem eigentlichen A-level (kurz A2 genannt) mit einer Prüfung am Ende des 13. Schuljahrs. Im Schuljahr 2016/2017 findet eine durchgreifende Reform des Lehrplans statt, so dass die 12. Klasse schon dem neuen Lehrplan folgt, während die 13. Klasse noch beim alten bleibt. Da es sich jedoch bei den Erfahrungen der Gewährspersonen um den alten Lehrplan handelt, haben wir es hier zunächst ausschließlich mit diesem zu tun. Im Abschnitt 7 werden jedoch die wesentlichsten Änderungen des reformierten Lehrplans besprochen, die einige anerkannte Schwächen des alten Lehrplans zu bewältigen suchen. 
Ein wesentliches Merkmal des englischen Bildungswesens ist der Umstand, dass für das A-level - also für die Prüfung zur Hochschulreife - normalerweise nur drei Fächer belegt werden, und im typischen Fall verfolgt man im Anschluss darauf ein Hochschulstudium in einem dieser Fächer. In England unterliegt die Zulassung zum Universitätsstudium einem sehr streng gehaltenen Numerus Clausus, mit der Folge, dass es eine intensive Konkurrenz um die Erlangung von Studienplätzen an einer ,guten“ Universität gibt, und die erfolgreiche Bewerbung hängt weitgehend von den Leistungen in den A-level-Prüfungen ab. Auch werden alle Schulen von Beamten des Kultusministeriums nach den Leistungen bei den GCSE- und A-level-Prüfungen bewertet und nach einer offiziellen Rangordnung gestuft (vgl. GOV.UK 2016). Ein bedeutendes Ergebnis davon ist der enorme Druck seitens der Schuldirektionen (sowie der Eltern) auf alle Lehrer, um bei ihren Schülern hohe Noten zu erzielen. In diesem Beitrag wird auch gezeigt, wie diese Struktur des Bildungswesens im sekundären und tertiären Bereich weitreichende (und vielleicht nicht beabsichtigte) Folgen für den Fremdsprachenunterricht an englischen Schulen hat und für den Erwerb eines vollen Spektrums von mündlichen und schriftlichen Kompetenzen in keinerlei Weise förderlich ist.

\section{Die A-level Prüfungen in Deutsch}

Wie im Abschnitt 2 erklärt wurde, bestehen die A-level Prüfungen in Deutsch bis zum Sommer 2017 aus zwei Teilen, und zwar dem AS-level, das nach dem 12. Schuljahr abgelegt wird, und dem $A 2$ im Jahr danach. Beide bestehen aus einem mündlichen und einem schriftlichen Teil, wobei ersterer in jedem der zwei Jahre mit 15\% der Gesamtnote bewertet wird und letzterer mit 35\%. Die Lehrpläne der verschiedenen Prüfungsbehörden unterscheiden sich geringfügig im Detail, 
aber alle entsprechen den absichtlich skizzenhaft gehaltenen Vorgaben des Ministeriums. Den Angaben in diesem Beitrag liegt der Lehrplan der größten Prüfungsbehörde AQA zu Grunde (vgl. AQA 2013), vor allem weil sich die meisten befragten Lehrerinnen für dieses Programm entschieden hatten, und es lag mir daher mehr Material darüber vor. Nach diesem Lehrplan wird die Fremdsprache in Bezug auf eine Anzahl von vorgegebenen Themen unterrichtet (vgl. AQA 2013: 5-7), und zwar für das 12. Schuljahr „Media“, „Popular Culture“, „Healthy Living/Lifestyle“ und „Family/Relationships“. Dazu kommen im 13. Schuljahr die Themen „Environment“, „The Multicultural Society“ und „Contemporary Social Issues“. Jedoch brauchen diese Themen nicht unbedingt im Kontext des Lebens oder der Kultur des jeweiligen Sprachgebiets studiert zu werden (vgl. AQA 2013: 5):

„As Knowledge of Society is not a discrete assessment objective in the subject criteria for Modern Foreign Languages, there is no requirement to study the topics in the context of the target language-speaking country/community. However, assessment material will include authentic sources.“

Im 13. Schuljahr werden zwei weitere frei gewählte Themen über die Kultur des betreffenden Sprachgebiets studiert, die von der Prüfungsbehörde auf Grund ihres vermeintlichen inhärenten Interesses für Jugendliche ausgewählt wurden. Diese dienen aber ausschließlich als Grundlage für die Entwicklung von sprachlichen Fähigkeiten und keineswegs zum Erwerb von etwaigen Stoffkenntnissen. Allerdings sind Zweifel darüber angebracht, ob diese Themen, die in der kurzen Zeit sowieso nur flüchtig behandelt werden können, tatsächlich das für sie beanspruchte Interesse seitens der Zielgruppe genießen sowie auch, ob ein solches Programm wirklich dazu geeignet ist, den Erwerb fremdsprachlicher 
Kenntnisse bei Jugendlichen zu fördern in einem Land, wo dies ohnehin problematisch ist. Außerdem gehört das zu Grunde gelegte Material ausschließlich dem Register des formellen Schrifttums an (auch wenn es sich um gesprochene Texte handelt), und die Lernenden werden kaum mit typischen Erscheinungen der alltäglichen Mündlichkeit konfrontiert.

Wie aus den begleitenden Kursheften klar wird, z.B. Niedziela u.a. (2008) für die 12. und Halstead u.a. (2009) für die 13. Klasse, sowie aus meinen eigenen Beobachtungen und Gesprächen mit Lehrerinnen und Studienanfängern, besteht der Unterricht weitgehend darin, Texte über die vorgeschriebenen Themen zu lesen oder zu hören, und es wird dann in der Klasse darüber diskutiert, oder es werden kurze schriftliche Arbeiten verfasst. Diesem Schema entspricht dann die Struktur der beiden Prüfungen am Ende des 12. und 13. Schuljahrs, die aus einem schriftlichen und einem mündlichen Teil bestehen ${ }^{6}$, wobei alle Teile auf Material mit einem Bezug zu den vorgeschriebenen Themen basieren.

In der mündlichen Prüfung, die in jedem der zwei Jahre 15\% der Gesamtnote ausmacht, werden den Schülern zunächst zwei Blätter mit jeweils zwei Stimulusfragen mit einem Bezug auf die studierten Themen gegeben. Sie müssen dann eines der Blätter wählen, und sie haben zwanzig Minuten, um eine Stellungnahme zu einer dieser Fragen vorzubereiten, die sie dann eine Minute lang vortragen sollen. Dieser kurze Vortrag bildet den Stoff zu einer Diskussion von etwa vier Minuten, worauf eine weitere Diskussion von etwa zehn Minuten über die beiden von dem Schüler gewählten „kulturellen“ Themen folgt. Es ist also klar, dass es sich in dieser Prüfung keineswegs um ein alltägliches mündliches Gespräch handelt, sondern um eine formelle Redesituation mit einem präparierten Vortrag und einem strukturierten 
Meinungsaustausch. Hier wird nicht die Fähigkeit zur fremdsprachlichen Kommunikation in typischen

Redesituationen gemessen, mit denen Lernende im Alltag im Sprachgebiet konfrontiert werden, sondern die Fähigkeit, für das formelle Schrifttum charakteristische sprachliche Formen auch mündlich wiederzugeben, unter genauer Beachtung der flexionsmorphologischen Normen und unter Verwendung von möglichst vielen unterschiedlichen grammatischen Konstruktionen und einem breiten auf das relevante Thema bezogenen Wortschatz. Nach den vorgeschriebenen Bewertungskriterien für die höchste Note in der mündlichen Prüfung soll der Kandidat nämlich Folgendes vorweisen (vgl. AQA 2013: 15): „Very good command of the language. Good use of idiom, complex structures and range of vocabulary. Highly accurate grammar and sentence structure; occasional mistakes.“ Dass diese Kriterien auch relativ vage sind (wie „occasional“ dürfen z.B. die „occasional mistakes“ sein) und dem Prüfer viel Raum zur eigenen Interpretation offen lassen, liegt auf der Hand, und die befragten Lehrerinnen haben häufig etwas Unsicherheit bzw. Skepsis geäußert, wie sie in der Tat gehandhabt werden könnten. Eine Lehrerin meinte z.B., sie gehe davon aus, dass die Prüfer eine „expectation that students should speak correctly“ hätten und sie daher Varianten im Unterricht meide, die für die Mündlichkeit typisch sind, obwohl es keine Hinweise dafür gibt, dass solche Formen tatsächlich negativ bewertet würden. Angesichts der Bedeutung der Noten für alle Beteiligten versteht es sich aber, dass die Lehrerinnen vielleicht zu Unrecht - eine gewisse Angst vor Variabilität bei der Bewertung haben und sich dadurch vor Risiken schützen, dass sie den Lernenden nur formelle hochsprachliche Normen beibringen.

Auch der Test zum Hörverständnis bewegt sich weitgehend im Bereich der konzeptionellen Schriftlichkeit. 
Von den vier Prüfungstexten aus dem Jahr $2015^{7}$ geht es in dreien um formelle Vorträge oder Diskussionen zu den vorgeschriebenen Themen, und zwar „Umweltlärm“, „Integrationsmaßnahmen“ und „Migration innerhalb der EU“. Beim vierten handelt es sich um ,ein Interview mit Alex, einem Häftling im Jugendarrest in Regensburg“. Bei diesem dürfte man vielleicht einen spontan gesprochenen Text mit charakteristischen Merkmalen der Mündlichkeit erwarten, eventuell mit regionalem Einschlag, aber er hört sich an wie ein langsam und sorgfältig vorgelesener Text, außerdem noch in perfektem, akzentfreiem Hochdeutsch. Mit der in einer solchen Situation tatsächlich zu erwartenden mündlich verwendeten Varietät des Deutschen hat dieser Text wenig gemeinsam und ist letztendlich so unwahrscheinlich, wie - hoffentlich - die Möglichkeit, dass die englischen Deutschlerner in eine derartige Situation in Deutschland geraten könnten.

\section{Bemerkungen zum Lehrplan für A-levels}

Nach diesen Ausführungen dürfte es klar sein, dass die Praxis des Deutschunterrichts in der Oberstufe englischer Schulen aus fremdsprachenpädagogischer Sicht einige problematische Aspekte hat. Man kann sich des Eindrucks nicht erwehren, dass diesem Lehrplan stillschweigend das Ziel zu Grunde liegt, auf eine möglichst einfache, mechanische und nicht anfechtbare Weise Leistungsmessungen im Endexamen $A$ level zu erzeugen, d.h. die Noten, die über die Zulassung zum Studium an bestimmten Universitäten entscheiden. Der Lehrplan hat einen engen Fokus auf das Studium einiger ausgewählter Themen, die nicht unbedingt einen Bezug zum Leben und zur Kultur des deutschsprachigen Raumes haben. Auch könnte man meinen, dass die Art und Weise, wie diese Themen in dem Lehrplan gehandhabt werden, das eigentliche Lernziel des Unterrichts verstellen, wenn man letzteres als 
den Erwerb von umfangreichen Kompetenzen in der Fremdsprache auffasst. Obwohl ausdrücklich darauf hingewiesen wird, dass stoffliche Kenntnisse nicht geprüft werden, sind solche klar erforderlich, um die Prüfung mit Erfolg abzulegen, und die Kenntnisse in der Fremdsprache, die man dabei erlangt, beschränken sich zwangsläufig auf die durch diese Themen gesetzten Rahmen. Nach den Bestimmungen der vom Kultusministerium mit der Aufrechterhaltung der Leistungsqualität bei offiziell anerkannten Examina beauftragten Behörde (das „Office of Qualifications and Examinations Regulation“ - kurz „Ofqual“" benannt, vgl. Ofqual 2011: 3), ist es ein Grundziel dieses Lehrplans, dass die Kandidaten „develop understanding of the language in a variety of contexts and genres” und „communicate confidently, clearly and effectively in the language for a range of purposes“, aber es ist fragwürdig, ob dieses Ziel je im Rahmen eines solchen beschränkten Lehrplans erfüllt werden kann. Auch erscheint es als bedenklich, dass das erzielte Niveau der fremdsprachlichen Kompetenzen in einer nationalen Prüfung zur Hochschulreife nicht mit den Bestimmungen des Gemeinsamen Europäischen Referenzrahmens in Bezug gebracht wird, der ein präziseres Bild der erlangten Kompetenzen abgeben würde als die äußerst vagen Angaben der Prüfungsbehörden, wie z.B. bei AQA (2013: 12-13), bei denen die Lehrerinnen Zweifel geäußert haben, ob sie sich überhaupt konsequent anwenden ließen.

Wenn man diese Prüfung als Ganzes in Betracht zieht, muss es als bedenklich gelten, dass die Kandidaten zur Erlangung einer Qualifikation zur Hochschulreife im Fach Deutsch in einer wenig umfangreichen Klausur lediglich einige relativ einfache Aufgaben ausführen müssen, von denen man kaum behaupten könnte, dass sie die sprachliche Kompetenz der Kandidaten in allen vier Fertigkeiten 
systematisch und ausführlich testen. Für viele Tests geht es ja in der Hauptsache um die gesteuerte Wiedergabe von auswendig gelernten sprachlichen Fragmenten - um „managed language reproduction“, wie ein Kollege das treffend bezeichnet hat - nicht um die systematische Messung echter fremdsprachlicher Fertigkeiten. Für das Kriterium, in den schriftlichen Tests ein ,very wide range of complex structures" zu gebrauchen, wurde von den befragten Studentinnen z.B. gemeldet, sie hätten eine Anzahl grammatischer Konstruktionen auswendig gelernt, die sie unbedingt in ihrem Aufsatz verwenden müssten, egal ob sie für das jeweilige Thema geeignet wären. Ähnliches gilt für den Wortschatz, wobei nach Angaben der Studenten anscheinend eindrucksvolle Redewendungen gelernt wurden, die sie in dem Aufsatz oder in der mündlichen Prüfung verwenden sollten. Als Beispiel hat eine Studierende den Ausdruck „meines Erachtens“ erwähnt, von dem sie sagte: „I learnt it and used it because the teacher told me it would impress the examiner, but when I went to Germany and used it everybody laughed at me because they thought it was funny and dreadfully stuck up“. Ihr wurde eben nicht erklärt, dass es sich um eine Wendung handelt, deren Gebrauch vorwiegend auf die formelle Schriftlichkeit beschränkt ist. Alle anderen studentischen Informantinnen meldeten ähnliche Erfahrungen.

Das Wunschziel englischer Deutschlehrer, wie sie es im Laufe meiner Befragungen geäußert haben, bestünde im Idealfall darin, den Schülern gute praktische Kompetenzen in der Fremdsprache beizubringen, und das würde auch den Vorstellungen und Erwartungen der Schüler entsprechen: Jedoch lassen sich diese Ziele mit einem solchen Lehrplan und diesen Tests kaum erfüllen. Eine Lehrerin meinte: „As much as we would like to teach them German for them to use, what we have to do first and foremost is to teach them to 
jump through hoops and pass the exam with good grades“. Damit die Schüler die für die Zulassung zu der angestrebten Universität erforderlichen Noten erlangen und die Schule ihre Position in der Rangliste des Bildungsministeriums aufrechterhält, haben die Lehrer eben keine andere Alternative, als die Schüler zielstrebig auf die beschränkten Erfordernisse des Examens hin zu drillen. Ob sie dabei breite und echt brauchbare fremdsprachliche Kompetenzen erwerben, hängt vom Zufall ab oder von der Fertigkeit der Lehrer, diese trotz der Beschränkungen des Lehrplans oder neben diesem her zu vermitteln.

\section{Zum Erwerb von Grammatik und Wortschatz im}

\section{Lehrplan für A-level}

Bedenklich erscheint es auch, dass in diesem Lehrplan eigentlich kein systematischer Sprachunterricht vorgesehen ist. Der Fokus des Unterrichts besteht vornehmlich in dem Studium einiger vorgeschriebener Themen, und während diese sehr konkret dargestellt werden, wird das Niveau der angestrebten Kompetenzen in der Fremdsprache in den Vorschriften des Bildungsministeriums keineswegs so formuliert, dass sich die Lehrer eine klare Vorstellung von den Erwartungen der Prüfungsbehörden machen können. In den Vorgaben der Prüfungsbehörde AQA (2013: 16) liest man, dass ,candidates are expected to have studied the grammatical system and structures of the language during their course. In the examination they are required to use actively and accurately grammar and structures appropriate to the tasks set, drawn from the following lists." Darauf folgt (vgl. AQA 2013: 17) eine Liste der grammatischen Elemente, die die Kandidaten beherrschen sollen, die eigentlich von dem Inhaltsverzeichnis einer beliebigen deutschen Grammatik hätte abgeschrieben werden können. Die 
Prüfungsbehörden stellen nämlich kein durchdachtes

Programm dar, das zu einer systematischen Progression beim

Erwerb fremdsprachlicher Kenntnisse führen könnte, sondern nur diese unkommentierte Liste.

In einem neuen Bericht zum Fremdsprachenunterricht des Teaching Schools Council ${ }^{8}$ schreibt Bauckham (2016: 10) zu diesem Thema:

Grammatical knowledge of a language is cumulative.

Sometimes, course books often overlook this and do not build knowledge consistently or cumulatively. In these cases pupils are simply confused because they are taught disjointed segments of grammar at different points and are not given the chance to build and consolidate a larger grammatical system.

Obwohl sich diese Aussage vornehmlich auf den Unterricht in der Mittelstufe (d.h. zum GCSE) richtet, ist es klar, dass sie auch für den besprochenen Lehrplan zum A-level und die begleitenden Kurshefte Niedziela u.a. (2008) und Halstead u.a. (2009) völlig zutrifft. Grammatische Elemente werden fragmentarisch in einer vollkommen ungeordneten Reihenfolge angeboten, ohne systematischen Bezug auf die Lesetexte der jeweiligen Einheit, ohne jegliches System und vor allem - wie Bauckham (2016: 10) klarmacht - ohne jede Vorstellung davon, wie wichtig die Progression und die ständige Verfestigung beim Erwerb der fremdsprachlichen Grammatik ist, sowie auch ohne jede Kenntnis von der für die Fremdsprachendidaktik äußerst bedeutenden Einsicht, dass grammatische Strukturen am effektivsten dadurch gelernt werden, wenn die Lernenden selbst zur Entdeckung grammatischer Strukturen und Regelmäßigkeiten angeregt werden (vgl. Ellis / Shintani 2014: 112).

Auch im Bereich des Wortschatzes ist dieser Lehrplan in vielerlei Hinsicht nicht zufriedenstellend. Zur 
diesbezüglichen fremdsprachenpädagogischen Praxis an englischen Schulen schreibt Bauckham (2016: 9): „There is some evidence that weak vocabulary teaching is a major part of the low standards achieved by many British pupils, and their often weak sense of progress.“ Er empfiehlt, dass ,,[the] vocabulary to be taught should be informed by frequency of occurrence in the language $[\ldots]$ ”. Dies scheint so offensichtlich, dass man es ausdrücklich nicht zu erwähnen bräuchte, aber dass Bauckham diesen Hinweis trotzdem für nötig hält, weist darauf hin, dass es sich hier um ein echtes Problem handelt. Die befragten Studentinnen meldeten nämlich, dass ihnen kaum Hinweise gegeben wurden, wie man Vokabular systematisch und aktiv erwirbt, sondern es wurde häufig erwartet, dass sie sich den nötigen Wortschatz einfach durch mehrmalige Begegnungen damit in Gesprächen oder Texten aneignen könnten. Bei eigenen Beobachtungen von sprachpraktischen Übungen an der Universität Manchester fiel auf, dass nicht wenige Studenten die Wörter Benzin und Seife nicht kannten Dieser Umstand ließe sich jedoch aus der Struktur des Lehrplans erklären, in dem vor allem der Wortschatz in den Bereichen der vorgeschriebenen Themen beherrscht werden muss. Und weil es sich bei diesen hauptsächlich um das Vokabular der Schriftlichkeit handelt, kann das zur Folge haben, dass die systematische Aneignung des typischen Wortschatzes der Mündlichkeit vernachlässigt wird. Das Ergebnis davon ist es dann, wie alle befragten Studierenden bestätigten, dass die Lernenden nicht in der Lage sind, einfache alltägliche Äußerungen in der Fremdsprache zu verstehen oder einfache alltägliche Aufgaben auszuführen.

\section{Mündlichkeit und Schriftlichkeit beim A-level}

Aus dem Vorhergehenden wird klar, dass sich der fremdsprachliche Unterricht in der Oberstufe englischer 
Schulen ausschließlich im Bereich der Schriftlichkeit bewegt.

Die für das Studium der vorgeschriebenen Themen zu

Grunde gelegten Texte mögen authentisch sein, aber auch

wenn sie gesprochen sind, sind sie durchweg

schreibsprachlich konzipiert und gehören ausschließlich dem

Register der formellen Schriftlichkeit an. Es geht letztendlich um „,mündlich realisierte Schriftsprache“ (vgl. Hirschfeld /

Rösler / Schramm 2016: 133), und mit der im Rahmen dieses Lehrplans vermittelten Sprache werden die Lerner nicht mit dem reellen sprachlichen Alltag im deutschen Sprachraum also mit der echten Mündlichkeit - konfrontiert. Es ist also keineswegs der Fall, dass die Kandidaten, wie es Ofqual (2011: 3) für für den Fremdsprachenunterricht bei A-level vorschreibt, lernen, „,[to] communicate confidently, clearly and effectively in the language for a range of purposes". Im Gegenteil, es scheint immer noch der Fall zu sein, dass, wie Barbour / Stevenson (1990: 2) schreiben,

It is a common experience for many foreign students of German to arrive for the first time in a 'German-speaking country' and discover to their horror that the language they encounter bears little resemblance to the German they learned at school or even at a higher level.

Dies entspricht genau der eigenen Erfahrung vor einem halben Jahrhundert nach einem traditionellen (wenn auch sehr intensiven) Unterricht nach der GrammatikÜbersetzungs-Methode, aber auch die im Herbst 2016 befragten Studierenden der Germanistik bestätigten, dass ihnen die alltägliche Kommunikation bei ihren ersten Besuchen in Deutschland sehr schwerfiel, sei es, wie oben erwähnt, dass sie unangemessen formelle Wendungen oder Konstruktionen in Alltagsgesprächen verwendeten, oder weil sie häufige Wörter nicht kannten. Dies hängt nicht unbedingt nur mit den oben dargestellten Mängeln beim Erwerb von Wortschatz zusammen, sondern auch weil viele im Alltag 
häufig vorkommende Wörter als „umgangssprachlich“ gelten und den Lernenden vorenthalten werden, wie z.B. gucken, klappen und kriegen (vgl. dazu Durrell 2012: 94). So bestätigten die befragten erstsemestrigen Studentinnen, sie hätten sie auch erst bei einem Deutschlandbesuch gehört. Studierende und Schüler, die noch nie in Deutschland gewesen waren, kannten diese Wörter nicht, auch waren ihnen andere Spezifika des informellen sprechsprachlichen Registers des Deutschennicht geläufig, wie z.B. die reduzierten Formen der Artikelwörter oder Pronomina und die Apokope bei Verbendungen (ich hab', sie hätt', usw.). Aus den Beobachtungen bei sprachpraktischen Übungen im Wintersemester 2016 ergab sich z.B., dass niemand darauf aufmerksam gemacht worden sei, dass es im gesprochenen Deutsch einen Unterschied zwischen dem unbestimmten Artikel (etwa: Haste 'n Buch gekauft?) und dem Zahlwort ein (etwa: Haste ein Buch gekauft?) gibt, und in Gesprächen im Laufe der beobachteten sprachpraktischen Übungen verwendeten die Studierenden weder reduzierte Formen der Pronomina noch apokopierte Verbendungen. Bei einem Rollenspiel („im Reisebüro“) sagte z.B. eine teilnehmende Studentin „Ich habe eine Reise gebucht“ anstatt der für die Mündlichkeit charakteristischen Form: „Ich hab“ 'ne Reise gebucht"،, und im darauffolgenden Interview meinte sie „Nobody has ever told me that people say anything different".

Es ist natürlich äußerst bedenklich und zugleich bedauerlich, dass beim Lehrplan für Deutsch als Fremdsprache an englischen Schulen die Mündlichkeit auf eine Weise vernachlässigt wird, dass Absolventen dieses Programms (nach durchschnittlich mindestens vier Jahren Deutschunterricht) nicht imstande sind, alltägliche Handlungen in einem deutschsprachigen Land durchzuführen oder an informellen Gesprächen mit gleichaltrigen deutschen 
Muttersprachlern problemlos teilzunehmen. Die befragten Studentinnen gaben an, dass sie, wenn sie in Deutschland waren, sich im Umgang mit dem alltäglichen gesprochenen Deutsch unsicher fühlten und dass es ihnen überhaupt an Selbstvertrauen beim Sprechen fehle. Nach unsystematischen Schätzungen von mir und meinen Kolleginnen im Wintersemester 2016 (sowie auch im Laufe der letzten 10 bis 15 Jahre) entspricht die Sprechkompetenz von Studienanfängern im Fach Deutsch, die für die Zulassung zum Studium in Manchester mindestens die Note B beim Alevel erreicht hatten, ungefähr dem Niveau A1 des Gemeinsamen Europäischen Referenzrahmens, wobei die Leistungen in den schriftlichen Kompetenzen, insbesondere im Leseverständnis, bedeutend höher liegen. Die Lernenden werden einfach nie mit der echten Alltagssprache und der Mündlichkeit konfrontiert, denn die Lehrpläne gehen von der Annahme von einer sprachlichen Homogenität aufgrund der Standardvarietät aus, die - wenn überhaupt - nur in der Schriftlichkeit existiert. Ohne Berücksichtigung der Mündlichkeit hat das Lernziel, die Kandidaten sollten „communicate confidently, clearly and effectively in the language for a range of purposes" (vgl. AQA 2013: 19), den Anschein einer Vortäuschung.

\section{Die reformierten Lehrpläne}

Wie im Abschnitt 2 erwähnt, wurden die Lehrpläne zum A-level ab September 2016 geändert. Danach soll die Prüfung nicht mehr aus zwei Teilen bestehen, sondern es wird eine einzige Prüfung am Ende der 13. Klasse abgelegt (vgl. Ofqual 2015). Für einige Fächer, u.a. die modernen und klassischen Fremdsprachen, wurden durchgreifende Reformen ins Auge gefasst, und dazu wurde für die betroffenen Fächer ein Ausschuss von Fachexperten - das ALevel Content Advisory Board $(A L C A B)^{9}$ - gebildet, um das 
Kultusministerium über Schwächen der bestehenden

Lehrpläne zu beraten und Reformen vorzuschlagen. $A L C A B$

legte seinen Bericht im Juli 2014 ab (vgl. ALCAB 2014), und darauf entwarf das Office of Qualifications and Examinations (Ofqual) Richtlinien, nach denen die Prüfungsbehörden detaillierte Lehrpläne herausarbeiten sollten (vgl. Ofqual 2014). Der dadurch zu Stande gekommene neue Lehrplan für Deutsch war dann ab September 2016 für den Unterricht verbindlich, und ihm folgten schon die 12 . Klassen, die ich im Laufe dieser Untersuchung im Oktober und November 2016 beobachtet habe.

Der Ausschuss stellte fünf hauptsächliche Schwächen des Lehrplans vor (vgl. ALCAB 2014: 2-3). Insbesondere wurden einige der studierten Themenbereiche als „dull and uninspiring" bezeichnet ${ }^{10}$, und er schlug vor, dass entgegen der bisherigen Praxis alle Themen auf das betreffende Sprachgebiet bezogen werden sollten. Auch kritisierte der Ausschuss, dass ,some awarding organisations advise examiners not to penalise grammatical mistakes in some parts of the written examination" und entsprechend empfahl er einen geringeren Grad an Toleranz bei sprachlichen Fehlern. Zusammenfassend war es ein zentrales Anliegen des Ausschusses, dass der Lehrplan neben der Erlangung erweiterter sprachlicher Kompetenzen in allen Fertigkeiten intellektuell anspruchsvoll und interessant sein sollte. Bedauerlicherweise hat Ofqual in seinen Richtlinien die Empfehlung eines systematischen Bezugs auf den Gemeinsamen Europäischen Referenzrahmen nicht beachtet. Der auf dieser Grundlage aufgestellte Lehrplan der Prüfungsbehörde AQA (vgl. AQA 2016) sieht das Studium von vier Themen vor, die alle einen Bezug auf den deutschen Sprachraum haben, und zwar „Aspects of German-speaking society“, „Artistic culture in the German-speaking world“, „Multiculturalism in German-speaking society“ und „Aspects 
of political life in German-speaking society“. Diese werden in Bezug auf Lese- und Hörverständnis in einer Klausur von 21/2 Stunden geprüft, die 50\% der Gesamtnote zählt. Das Format dieser Prüfung unterscheidet sich im Grunde genommen nicht wesentlich von dem bisherigen. Zweitens werden in einer zweistündigen Klausur (20\% der Gesamtnote) zwei Aufsätze von ca. 300 Wörtern über ein Buch oder einen Film (bzw. über zwei Bücher) geschrieben, die zur Prüfung studiert wurden. Eine Liste der vorgeschriebenen Bücher und Filme für die Prüfung im Sommer 2018 sieht man bei AQA (2016: 18), sie enthält z.B. Max Frisch Andorra, Heines Buch der Lieder und Jana Hensel Zonenkinder, und als Filme Das Leben der Anderen und Good bye Lenin! Schließlich gibt es eine mündliche Prüfung (30\% der Gesamtnote) mit zunächst, wie in der bisherigen Prüfung, einer Diskussion über kurz vor der Prüfung ausgehändigten Stimulusfragen mit einem Bezug auf die studierten Themen. Dem folgt eine längere Diskussion über ein Forschungsprojekt zur Gesellschaft, Geschichte oder Kultur eines deutschsprachigen Landes, das der Kandidat eigens ausgewählt und unternommen hat.

Es ist klar, dass in diesem neuen Lehrplan einige Schwächen des bisherigen Programms beseitigt wurden, vor allem was den Anspruch und die Konsequenz der Themen betrifft, und es gibt den Lehrern viel größere Möglichkeiten, den Unterricht interessanter zu gestalten. In Bezug auf den Erwerb von sprachlichen Kompetenzen sind die Änderungen jedoch viel weniger durchgreifend. Wie schon erwähnt, wurde der Lehrplan relativ kurzfristig eingeführt, so dass mir im Moment Begleithefte nur für den Unterricht in der 12. Klasse vorlagen, noch nicht für die 13. Klasse. Das Format von diesen, z.B. Sauer u.a. (2016), gleicht völlig denen für den bisherigen Lehrplan, wie z.B. Niedziela u.a. (2008) und Halstead u.a. (2009), indem auch hier Elemente der 
Grammatik kapitelweise fragmentarisch angeboten werden, ohne auf Progression zu achten und ohne systematischen Bezug zur Sprache der Texte im jeweiligen Kapitel herzustellen. Die grundliegende Einsicht, dass grammatische Kenntnisse systematisch aufgebaut und entwickelt werden können, scheint den Autoren dieser Hefte entgangen zu sein. Zum Schluss bleiben alle Aspekte dieses Lehrplans sowie auch der Prüfungen trotz des Gebrauchs von authentischen Materialien und der vorgegebenen Lernziele der Prüfungsbehörde fest in der Schriftlichkeit verhaftet. Das sprachliche Register des Alltags, so wie es in der informellen Kommunikation zwischen Muttersprachlern vorkommt, wird nach wie vor ignoriert bzw. verschwiegen (fast als ob es sich um ein schmutziges Geheimnis handeln würde), und die grundverschiedene Eigenart der Mündlichkeit bleibt immer noch außer Betracht. Schon vor 20 Jahren schrieb McDonough (1995: 120): „We do not want to create a new breed of skilled test-takers - rather a generation of good learners who can demonstrate their knowledge and skills in valid language tests.“. In England scheint man in der Zwischenzeit nicht viel weiter gekommen zu sein.

\section{Literatur}

ALCAB (2014): Report of the Alcab Panel for Modern Foreign and Classical Languages. https://alevelcontent.files.wordpress.com/2014/07/alcabreport-of-panel-on-modern-foreign-and-classicallanguages-july-2014.pdf (letzter Zugriff 15. Januar 2017). AQA (2013): GCE AS and A Level Specification, French/German/Spanish. For exams from June 2014 onwards. For certification from June 2014 onwards. http://filestore.aqa.org.uk/subjects/specifications/alevel/A QA-2650-2660-2695-W-SP-14.PDF (letzter Zugriff 15. Januar 2017). 
AQA (2016): A-level German (7662). Specification. For teaching from September 2016 onwards. For exams in 2018 onwards.

http://filestore.aqa.org.uk/resources/german/specifications/ AQA-7662-SP-2016.PDF (letzter Zugriff 15. Januar 2017).

Barbour, J. Stephen / Stevenson, Patrick (1990): Variation in German. A Critical Approach to German Sociolinguistics. Cambridge.

Bauckham, Ian (2016): Modern Foreign Languages Pedagogy Review. A review of moderrn foreign languages teaching practice in key stage 3 and key stage 4 . http://tscouncil.org.uk/wp-content/uploads/2016/11/MFLPedagogy-Review-Report-2.pdf (letzter Zugriff 15. Januar 2017).

DfE (2013): National Curriculum in England: Languages Programmes of Study. https://www.gov.uk/government/publications/nationalcurriculum-in-england-languages-progammes-of-study (letzter Zugriff 15. Januar 2017).

Durrell, Martin (2012): Zur Relativierung von hochsprachlichen Normen in der deutschen Sprache der Gegenwart. Der Blick von außen. In: S. Günthner / W. Imo / D. Meer / J.G. Schneider (Hg.), Kommunikation und Öffentlichkeit. Sprachwissenschaftliche Potenziale zwischen Empirie und Norm. Berlin u. Boston, 85-101. Ellis, Rod / Shintani, Natsuko (2014): Exploring Language Pedagogy through Second Language Acquisition Research. London u. New York.

GOV.UK (2016): Check school performance tables. https://www.gov.uk/school-performance-tables (letzter Zugriff 15. Januar 2017).

Halstead, Peter / McNeill, Jeannie / Sandry, Claire / Shannon, Paul (2009): AQA German. A2. Cheltenham. 
Hirschfeld, Ursula / Rösler, Dietmar / Schramm, Karen (2016): Facetten der Mündlichkeit im DaF-Unterricht. Zur Einführung in den Themenschwerpunkt. In: Deutsch als Fremdsprache 53, 131-133.

McDonagh, Steven H. (1995): Strategy and Skill in Learning a Foreign Language London.

Niedziela, Monika / Shannon, Paul / Wardle, Michael (2008): AQA German AS. Cheltenham.

Ofqual (2011): GCE AS and A Level Subject Criteria for Modern Foreign Languages (MFL). https://www.gov.uk/government/publications/gce-as-anda-level-subject-criteria-for-modern-foreign-languages-mfl (letzter Zugriff 15. Januar 2017).

Ofqual (2014): Developing new GCSE, A level and AS qualifications for first teaching in 2016. https://www.gov.uk/government/uploads/system/uploads/a ttachment_data/file/377851/2014-07-15-developing-newgcse-a-level-and-as-qualifications-for-first-teaching-in.pdf (letzter Zugriff 15. Januar 2017).

Ofqual (2015): Guidance. Get the facts: AS and A level reform. https://www.gov.uk/government/publications/getthe-facts-gcse-and-a-level-reform/get-the-facts-as-and-alevel-reform (letzter Zugriff 15. Januar 2017).

Rösler, Dietmar (2016): Nähe und Distanz zur Mündlichkeit in der fremdsprachendidaktischen Diskussion. Versuch einer Annäherung. In: Deutsch als Fremdsprache 53, 135149.

Sauer, Dagmar / Schicker, Corinna / Sydenham, Keith / Klingler, Erika (2016): AQA German. A level Year 1 and AS. Oxford.

\footnotetext{
${ }^{1}$ In diesem Beitrag beziehen sich "England“ und „englisch" ausschließlich auf dieses Teil des Vereinigten Königreichs, weil das
} 
Bildungswesen in den anderen Landesteilen (Wales, Schottland, Nordirland) unterschiedlich strukturiert ist.

${ }^{2}$ Hier muss ich allen Informantinnen, Lernenden sowie auch Lehrenden, die mir bei dieser Untersuchung - unter der Bedingung der strikten Anonymität - als Informantinnen gedient haben, meinen aufrichtigen Dank aussprechen für ihre selbstlose und zuvorkommende Hilfe. Ich trage jedoch selber die alleinige Verantwortung für die hier vorgetragenen Ansichten.

Weil es sich bei den Informantinnen fast ausschließlich um Frauen gehandelt hat, wird in diesem Beitrag grundsätzlich nur die weibliche Form der relevanten Bezeichnungen verwendet. Nur bei Generalisierungen wird dann von dem Plural der männlichen Form (z.B. die Lehrer) Gebrauch gemacht.

${ }^{3}$ In diesem Zusammenhang bin ich den KollegInnen Katrin Kohl (Oxford), Stephen Parker (Manchester) und Jocelyn Wyburd (Cambridge) sehr zu Dank verpflichtet.

${ }^{4}$ Die ausführlichste und aktuellste Information über die GCSE-Prüfungen ist bei Wikipedia zu finden: https://en.wikipedia.org/wiki/General_Certificate_of_Secondary_Educat ion (letzter Zugriff 15. Januar 2017)

${ }^{5}$ Zuverlässige aktuelle Information über diese Prüfungsbehörden findet man ebenfalls bei Wikipedia:

https://en.wikipedia.org/wiki/Examination_boards_in_the_United_King dom\#Creation_of_the_current_boards (letzter Zugriff 15. Januar 2017)

${ }^{6}$ Die Prüfungen (einschl. der gesprochenen Texte zum Hörverständnis) für die Jahre 2013 bis 2015 kann man von der Webseite der AQA herunterladen: http://www.aqa.org.uk/exams-administration/examsguidance/find-past-papers-and-mark-schemes (letzter Zugriff 15. Januar 2017). Die Angaben in diesem Beitrag beziehen sich auf die Prüfungen im Sommer 2015.

${ }^{7}$ Downloadbar von http://filestore.aqa.org.uk/subjects/mp3/AQA26603-JUN15.MP3 (letzter Zugriff 15. Januar 2017)

${ }^{8}$ Zu diesem Lehrerverband vgl. http://tscouncil.org.uk/ (letzter Zugriff 15. Januar 2017)

${ }^{9}$ Informationen über das A-Level Content Advisory Board findet man auf der Webseite: https://alcab.org.uk/ (letzter Zugriff 15. Januar 2017).

${ }^{10}$ Dies wurde von zwei der befragten Studentinnen ausdrücklich bestätigt, die einige Themen als "dire“ bezeichneten. 